\title{
Article
}

\section{Yo-Yo Inspired Triboelectric Nanogenerator}

\author{
Deokjae Heo ${ }^{\dagger}$, Jihoon Chung ${ }^{\dagger}$, Gunsub Shin, Minhyeong Seok, Chanhee Lee and Sangmin Lee *
}

Citation: Heo, D.; Chung, J.; Shin, G.; Seok, M.; Lee, C.; Lee, S. Yo-Yo Inspired Triboelectric Nanogenerator. Energies 2021, 14, 1798. https:// doi.org/10.3390/en14071798

Academic Editors: Ya Yang and Minbaek Lee

Received: 5 February 2021

Accepted: 23 March 2021

Published: 24 March 2021

Publisher's Note: MDPI stays neutral with regard to jurisdictional claims in published maps and institutional affiliations.

Copyright: (C) 2021 by the authors. Licensee MDPI, Basel, Switzerland. This article is an open access article distributed under the terms and conditions of the Creative Commons Attribution (CC BY) license (https:// creativecommons.org/licenses/by/ $4.0 /)$.
School of Mechanical Engineering, Chung-Ang University, 84, Heukseok-ro, Dongjak-gu, Seoul 06974, Korea; ejrwo472@naver.com (D.H.); Jihoon@cau.ac.kr (J.C.); gunsub93@gmail.com (G.S.); dkshktpq@naver.com (M.S.); cksgm19507@gmail.com (C.L.)

* Correspondence: slee98@cau.ac.kr; Tel.: +82-2-820-5071

+ These authors contributed equally to this work.

Abstract: Recently, as the demand for sustainable and renewable energy to power a large number of small electronics and sensors has increased, various mechanical energy harvesters such as electromagnetic, piezoelectric, and triboelectric generators have been highlighted because they have no environmental constraints to generate electricity and function as sustainable power sources. Among these generators, triboelectric nanogenerators (TENGs), which produce electrical energy via triboelectrification and electrostatic induction, are a promising energy harvesting technology that can utilize existing materials or the structure of existing commercial products. Considering the vast number of independent portable electronics used today, the development of hand-driven TENGs is important. There is great demand for TENG considering both commercial product-inspired designs, which are the merit of TENG itself, and the hand-driven type. However, relevant studies are still lacking, and therefore further studies in these areas are required. In this study, we developed a novel triboelectric nanogenerator (Y-TENG) inspired by the Yo-Yo that can produce a sustainable electric output by hand motion input. One generator of Y-TENG produced a maximum $V_{O C}$ of $10 \mathrm{~V}$ and an $I_{C C}$ of $0.7 \mu \mathrm{A}$. Peak/root mean square (RMS) voltage output-based quantitative analysis for the optimized number of blades and dielectric material was performed. The proposed Y-TENG was able to continuously light up three light-emitting diodes (LEDs) while the Y-TENG moved up and down.

Keywords: energy harvesting; triboelectric nanogenerator; Yo-Yo; hand-driven device; mechanical energy

\section{Introduction}

With the advancement of the Internet of Things (IoT), the demand for renewable and sustainable energy sources to power small electronics and sensors is increasing. Accordingly, various electricity generation devices such as solar [1,2], thermoelectric [3,4], and radio frequency $[5,6]$ energy harvesters have been developed to function as auxiliary power sources. Among these electric generators, several mechanical energy harvesters using piezoelectric [7,8], electromagnetic [9,10], and triboelectric [11-14] effects are drawing attention as sustainable power sources because they have no environmental constraints to generate electricity. Triboelectric nanogenerators (TENGs), which convert mechanical energy into electrical energy by a combination of triboelectrification and electrostatic induction, are a prospective energy harvesting technology. Unlike other energy generation methods, TENGs have distinct advantages, including broad material availability, low cost, and high efficiency, and thus can utilize existing materials or structures of commercial products $[15,16]$. These TENGs tend to produce high electrical output with fast mechanical input $[17,18]$, whereas many other TENGs require additional automatic input sources such as high-speed actuators or motors to power small electronics $[19,20]$. Considering the vast number of independent portable electronic devices used today, the development of portable TENGs is important, as they can charge or supply power when needed without any constraints on the operating environment. In this regard, recent studies have focused 
on various TENGs driven by hand motion input [21,22]. In particular, there is great demand for TENGs that consider both commercial product-inspired designs, which is a merit of TENGs, and hand-driven types of TENGs, such as gyroscopes [23] and whirligig toys [24]. However, relevant studies are lacking, and further studies in these areas should be conducted. Herein, we developed a Yo-Yo-inspired triboelectric nanogenerator (Y-TENG), which is the first report on such a TENG. The Y-TENG consists of two generators, each of which is composed of a case, a shaft, and a blade inside the case. A dielectric layer (polytetrafluoroethylene, PTFE) and an electrode layer (Al) are attached to both sides of the blade and the inner surfaces of the case, respectively. When the Y-TENG is released downward by the force of a snap throw and gravity, the string looped around the shaft unwinds, and the case fixed to the shaft rotates. Subsequently, the blade follows the case and starts to rotate because of a bearing between the shaft and the blade. This relative motion between the case and blade generates an electrical output of high frequency. To optimize the design of the Y-TENG, the electrical output depending on the number of wings and material of the dielectric layer was measured. Considering the peak and root mean square (RMS) voltage, a quantitative analysis was performed in terms of the dynamic performance of the TENG. One generator of the fabricated Y-TENG, which was connected to a full-wave rectifier, lit up three light-emitting diodes (LEDs) continuously when the Y-TENG dropped and rose.

\section{Materials and Methods}

\subsection{Fabrication of $Y$-TENG}

Two cylindrical cases and one blade inside the cases combine to form one generator of the Y-TENG. Each case and blade is made of acrylonitrile butadiene styrene (ABS) as a substrate, and is fabricated by $3 \mathrm{D}$ printing. The surface of each case has a circular shape with four hollow segments with a radius of $4 \mathrm{~cm}$ located at intervals of $45^{\circ}$. The blade also has a four-sector structure with the same radius of $4 \mathrm{~cm}$ and the sectors are located at intervals of $45^{\circ}$. Commercial aluminum tape with a thickness of $0.05 \mathrm{~mm}$ (DUCKSUNG Co., Seoul, Korea) is attached on the two inner surfaces of the case, and commercial PTFE tape with a thickness of $0.08 \mathrm{~mm}$ (CHUKOH Co., Bangkok, Thailand) is attached to both sides of the blade, which is tailored into the same four-segment shape. The Al electrode and PTFE dielectric layer are separated by a very small gap of $1 \mathrm{~mm}$, but are not in contact, and are parallel to each other. A commercial shaft (PSSFUL3-25-B5-S5, MISUMI Co., Schaumburg, IL, USA) was inserted into the center of each of the two generators and fixed in place. A commercial bearing (693zz, BEARING STORE Co., Seoul, Korea) was inserted between the blade and shaft. Photographs of the fabricated Y-TENG are shown in Supplementary Materials Figure S3. A string is looped around the center of the shaft, similar to a spool.

\subsection{Electrical Measurement}

The voltage and current were measured using an oscilloscope (MDO 3014, Tektronix Co., Beaverton, OR, USA) and a current preamplifier (MODEL SR570, Stanford Research Systems Co., Sunnyvale, CA, USA).

\section{Results and Discussion}

Figure 1a shows a schematic illustration of the Y-TENG, which consists of two generators on the left and right sides. Each generator is composed of three basic components: a central shaft, a cylindrical case fixed to the shaft, and a freestanding blade inside the case. The cylindrical case and the blade are made of acrylonitrile butadiene styrene (ABS), which was used as a substrate for coating with various polymers. Each circular surface of the cylindrical case has four segments that are alternately positioned. The freestanding blade also has a four-sector structure. Each aluminum (Al) electrode and polytetrafluoroethylene (PTFE) dielectric layer tailored into the same four-segment shape is attached to the inner surfaces of the cylindrical case and both sides of the freestanding blade. The Al electrode and PTFE dielectric layer are separated by a very small gap of $1 \mathrm{~mm}$ but are not in contact 
and are parallel to each other. In the Yo-Yo design, the Y-TENG has a string looped around the shaft, similar to a spool. When the free end of the string is held by a finger and Y-TENG is released downward by gravity and the force of a snap throw, the string is unwound, and both the cylindrical case and the blade of Y-TENG start to rotate. Then, relative motion occurs between the cylindrical case and the blade because there is a bearing between the shaft and the freestanding blade. The cylindrical case which has the same mechanical rotation motion with the shaft rotates first, and then the freestanding blade follows to rotate by friction of the bearing.

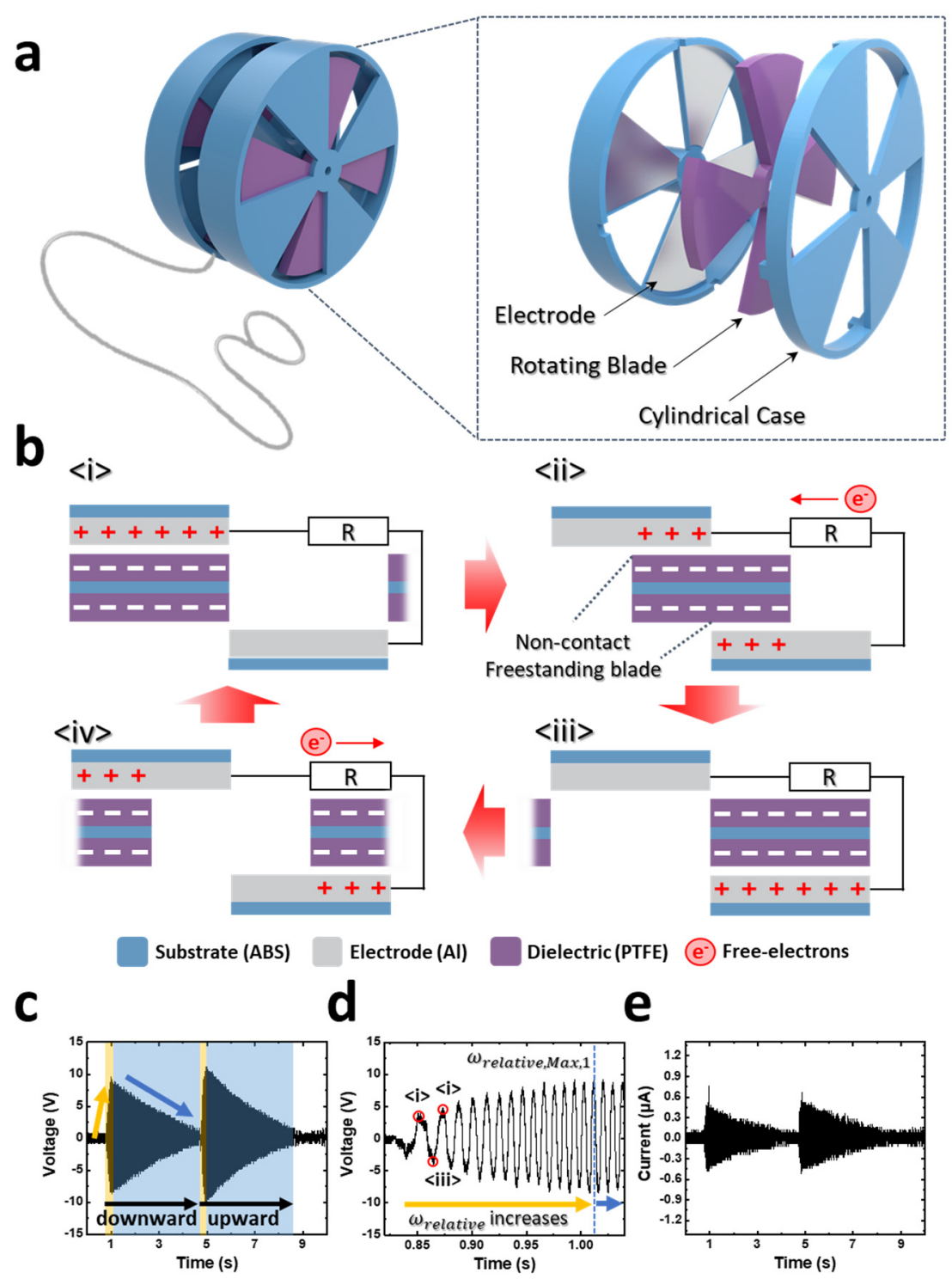

Figure 1. Yo-Yo-inspired triboelectric nanogenerator (Y-TENG) (a) Schematic illustration of Y-TENG; (b) working mechanism for electricity generation; (c) voltage output plot; (d) magnified voltage output plot corresponding to the first yellow region in Figure 1c; (e) current output plot.

The detailed working mechanism for electricity generation by the Y-TENG is schematically illustrated in Figure 1b, based on a single generator. The two upper and lower Al electrodes on the inner surface of the substrate case are electrically connected to function as freestanding TENGs [25]. The two PTFE layers attached to both sides of the substrate blade do not affect the generator on the other side. The surface of the PTFE dielectric layer has a negative charge owing to its high electron affinity, and additional triboelectric negative charges can be formed via pre-charging with fur. As the string is unwound, relative motion 
occurs between the cylindrical case and the blade. First, when the blade is aligned with the upper $\mathrm{Al}$ electrode, the negative surface charge of the blade induces positive charges on the upper Al electrode (Figure 1bi). As the blade moves and is located near the lower $\mathrm{Al}$ electrode, free electrons flow from the lower $\mathrm{Al}$ electrode to the upper $\mathrm{Al}$ electrode, and positive charges are fully induced on the lower $\mathrm{Al}$ electrode by the negative charge of the blade surface (Figure 1bi-iii). Furthermore, while the blade passes over the lower Al electrode and the next blade is positioned at the upper $\mathrm{Al}$ electrode, free electrons flow back from the upper $\mathrm{Al}$ electrode to the lower $\mathrm{Al}$ electrode, and positive charges are induced on the upper Al electrode by the negative surface charge of the blade (Figure 1bi-iii).

Based on one generator of the Y-TENG, when the Y-TENG with the blade containing four wings is released downward and returns upward, an electrical output of high frequency is generated continuously. As shown in the corresponding peak open-circuit voltage $\left(V_{O C}\right)$ output (Figure 1c), when the Y-TENG moves downward, the electrical output increases rapidly (yellow region) and decreases slowly (blue region). This process is repeated when the Y-TENG moves upward. Figure 1e shows the corresponding peak closed-circuit current output $\left(I_{C C}\right)$ of the Y-TENG. The maximum $V_{O C}$ and $I_{C C}$ generated by the Y-TENG are $10 \mathrm{~V}$ and $0.7 \mu \mathrm{A}$, respectively. Figure $1 \mathrm{~d}$ shows a magnified output plot that corresponds to the first yellow region in Figure 1c. The electrical output of the Y-TENG has a continuous alternating peak waveform, and each local maximum and local minimum point (red circle) of one peak corresponds to Figure 1bi,biii, respectively. The peak value increases as the relative rotational velocity between the cylindrical case and blade $\left(\omega_{\text {relative }}\right)$ increases (yellow region). After the peak value reaches the first maximum $\left(\omega_{\text {relative,Max }, 1}\right)$, blue dotted line), the peak value decreases as $\omega_{\text {relative }}$ decreases. This tendency is based on previous rotational TENG studies, in which electrical output increases depending on the rotating speed of the rotator because the increase of approaching and separating rate between two surfaces induces more charges within the same time frame $[19,20,26]$.

To elaborate on and analyze this result in terms of dynamics, several assumptions were made for simplification. First, the Y-TENG case-shaft system used in this study is based on a Yo-Yo which moves downward and returns at a constant linear and rotational velocity when no additional snap throwing or pulling force is applied. This is because of the no-slip condition between the string and shaft and the force equilibrium between tension and gravity. Second, constant kinetic friction is applied to the blade by a bearing that is positioned between the blade and the shaft-case system and induces a constant rotational acceleration of the blade.

In other words, the relative rotational motion between the case and blade is directly connected to the electrical output of the Y-TENG. Figure S1a shows a simplified scheme of the Y-TENG rotating on the center of mass (COM) of the case with relative rotational velocity $\left(\omega_{\text {relative }}\right)$. From the perspective of the rotating case (Figure S1b), when the YTENG is released downward by a snap throw and gravity, the constant snap throwing force increases the rotational velocity of the case $\left(\omega_{\text {Case }}\right)$ linearly for a moment, which induces additional tension (Figure S1bi). Subsequently, when no snap throwing force is applied, the case spins and moves down at a constant velocity (Figure S1bii). The case reaches a low point and maintains rotational energy at the same velocity by rotational inertia (Figure S1biii). When the case returns upward by the inertia of rotation and the string starts to wind itself back up, a constant pulling force is applied to the case by the string. The pulling force also instantaneously boosts the velocity of the case in a linear relationship (Figure S1biv). Finally, when no pulling force acts, the case returns to the initial position with a constant velocity (Figure S1bv). From the point of view of the blade (Figure S1c), while the case-shaft system is released and returned, the blade $\left(\omega_{\text {Blade }}\right)$ is assumed to have uniformly accelerated motion by constant kinetic friction of the bearing. Figure S1d shows a plot of the rotational velocity versus time, consisting of red and purple lines. Each of the steps "" to "v" corresponds to the description in Figure S1b. The red line represents the rotational velocity of the case $\left(\omega_{\text {Case }}\right)$, which goes through uniform acceleration, uniform velocity, uniform acceleration, and uniform velocity. The purple 
line represents the rotational velocity of the blade $\left(\omega_{\text {Blade }}\right)$, which undergoes uniformly accelerated motion. Figure S1e shows a plot of the relative rotational velocity $\left(\omega_{\text {relative }}\right)$ versus time, which corresponds to the relative difference between $\omega_{\text {Case }}$ and $\omega_{\text {Blade }}$ in Figure S1d. As shown in the plot, the $\omega_{\text {relative }}$ increases rapidly at step " $i$ " and decreases slowly from step "ii" to "iii", and then this process is repeated from steps "iv" and "v". This waveform variation corresponds to the electrical output plot of the Y-TENG in Figure 1c-d and occurs because the relative rotational velocity between the case and blade directly affects the electrical output of the Y-TENG.

The number of wings that the blade has can affect the electrical performance of the Y-TENG. Figure 2a shows a schematic illustration of case-blade sets having 1, 2, 3, or 4 wings, where the wings are separated by $180^{\circ}, 90^{\circ}, 60^{\circ}$, or $45^{\circ}$, respectively. Each case has the same number of segments as the number of wings on the blade. Figure $2 b$ shows the $V_{O C}$ output of the Y-TENG depending on the number of wings. The Y-TENG with four wings generated the highest peak voltage of 20-30 V. The Y-TENGs with one to three wings showed a peak voltage between 10 and $20 \mathrm{~V}$. However, the output waveform generated by the Y-TENG exhibits an extremely sharp peak. Therefore, it is necessary to convert the peak voltage to the root mean square voltage $\left(V_{R M S}\right)$, which is a better quantitative indicator than the peak voltage. The $V_{R M S}$ was calculated from the following equation:

$$
V_{R M S}=\sqrt{\frac{\int V(t)^{2} \mathrm{~d} t}{T}}
$$

where $V(t)$ and $T$ denote the peak voltage output and the measurement period, respectively. It is noted that the $V_{R M S}$ output of the Y-TENG increased from one to four wings, and the Y-TENG with four wings produced the highest $V_{R M S}$ output of $5 \mathrm{~V}$ (Figure 2c).

a

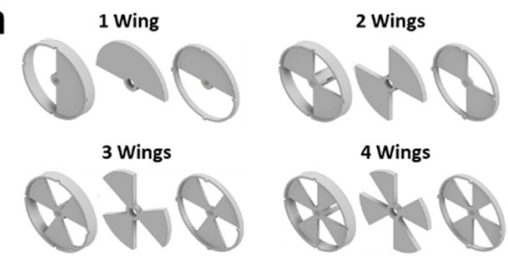

b

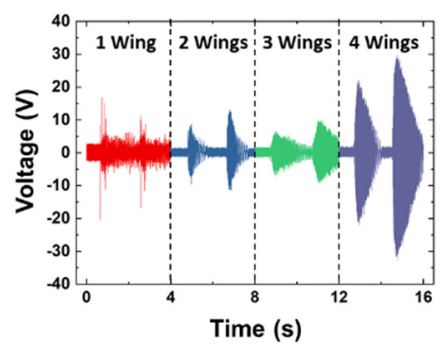

C

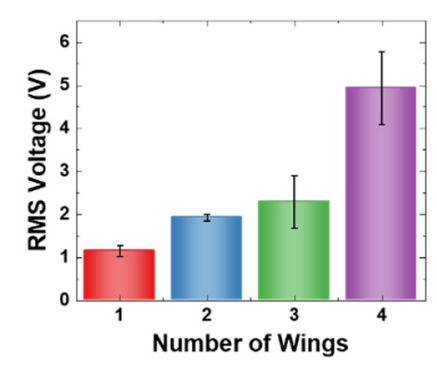

Figure 2. Electrical performance of the Y-TENG for different number of wings. (a) Schematic illustration of case-blade sets having 1, 2, 3, or 4 wings; (b) peak voltage and (c) root mean square (RMS) voltage output of the Y-TENG depending on the number of wings. 
In terms of dynamics, if we assume that there is no difference in motion of the case according to the number of segments, and the same constant kinetic friction is applied to the blade regardless of the number of blades, each blade with one to four wings has different mechanical behavior. As shown in Figure S2, the blade with one wing is initially located at the bottom because the mass of the blade is concentrated on one $180^{\circ}$ wing, and because of rotational movement by gravity, which occurs naturally and causes the wing to descend. It is difficult to overcome gravity and raise one wing for rotation owing to the small amount of kinetic friction at the bearing. In the case of two to four wings, even if a small amount of bearing kinetic friction is applied, it is relatively easy to overcome gravity and rotate the wings because the mass of the blade is distributed over multiple wings. This means that the blade with four wings is the easiest to rotate and has the smallest relative rotational velocity with the rotating case. However, this expected trend does not agree with the electrical output result in Figure 2a,b. It is expected that an increase in the number of wings will have a surface patterning effect and that this would more dominantly influence the electrical output of the Y-TENG than the effect of mechanical movement. In general, as the number of surface patterns between the triboelectric layer and electrode increases, the electrical output of TENGs tends to increase [27].

Figure 3 a shows the $V_{O C}$ output of the Y-TENG depending on the triboelectric material of the layers attached to both sides of the substrate blade. As shown in the plot, the maximum peak voltage generated by each triboelectric material is similar. Y-TENGs having blades coated with polytetrafluoroethylene (PTFE), polyurethane (PU), polyimide (PI), or nylon show peak voltages of 20 to $25 \mathrm{~V}$. Only the Y-TENG with ABS blades shows a peak voltage under $15 \mathrm{~V}$. However, the $V_{R M S}$ output of each material shows different results. As shown in the $V_{R M S}$ plot (Figure $3 \mathrm{~b}$ ), the Y-TENG with the PTFE-coated blade exhibits the highest $V_{R M S}$ output of $2.4 \mathrm{~V}$. This is due to the high surface charge of PTFE and high electron affinity, generating a higher electrical output compared to other materials [28]. PTFE was therefore chosen as the blade surface to generate a high electrical output.

\section{a}

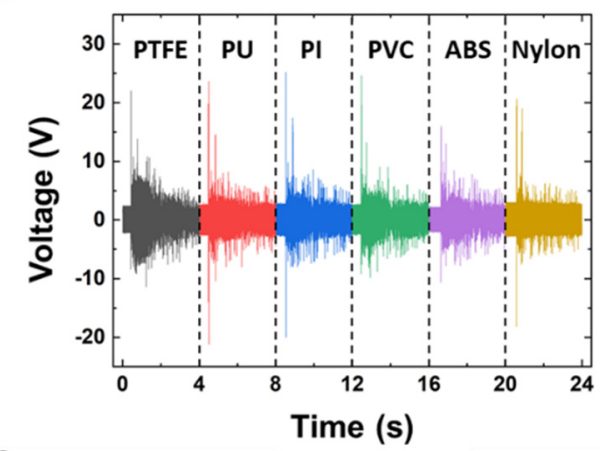

b

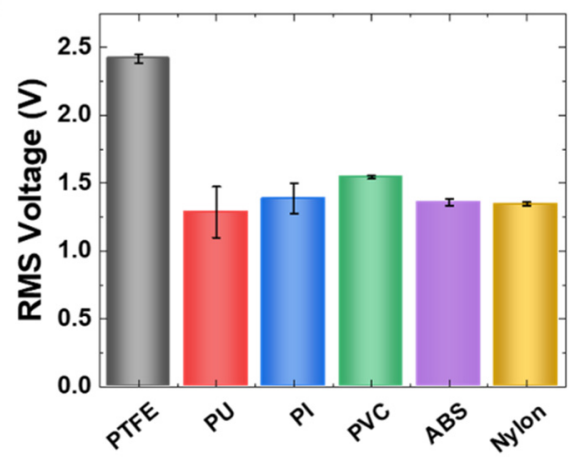

Figure 3. Electrical performance of Y-TENG for different materials of triboelectric layer. (a) Peak voltage and (b) RMS voltage output of the Y-TENG depending on various triboelectric materials. 
Figure 4a shows the Y-TENG connected to a rectifying circuit to light up an LED array during operation. As shown in Figure S3, the Y-TENG consists of two generators on the left and right sides. Each generator is connected to a full-wave rectifier and three LEDs to demonstrate power generation when the operator drops the Y-TENG. As shown in Figure $4 \mathrm{~b}$, the LED array lights up as the Y-TENG drops and rises. The inset photograph in Figure $4 \mathrm{~b}$ shows three LED lights during rotation.

a

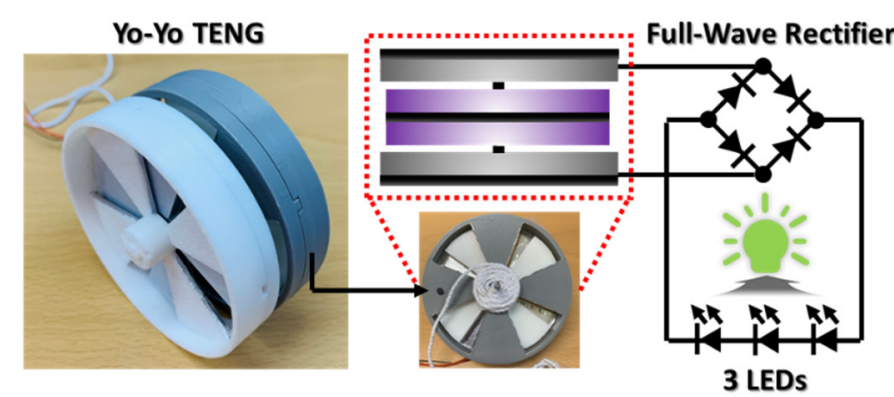

b

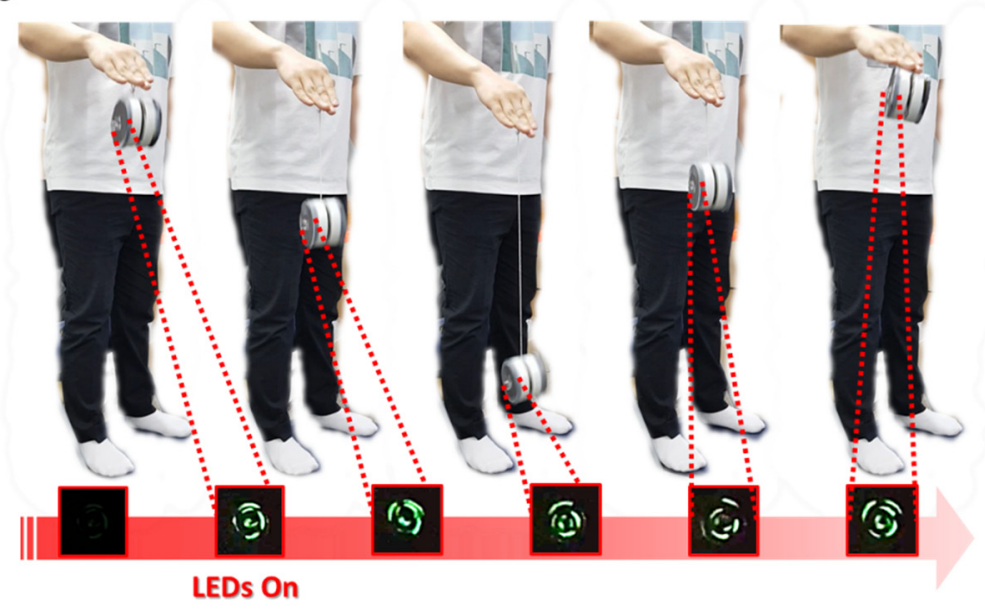

Figure 4. Demonstration of power generation when the operator drops the Y-TENG. (a) Y-TENG connected to a rectifying circuit to light up an LED array during operation; (b) the LED array lights up as the Y-TENG drops and rises.

Furthermore, voltage and power output according to various external load resistances are measured (Figure S4). Considering general characteristics of TENGs which has extremely high internal impedance as much as tens of mega ohm [29], voltage output of Y-TENG is measured under external load resistance of $500 \mathrm{k} \Omega$ to $300 \mathrm{M} \Omega$. Power output is calculated from the equation of Power $(\mathrm{W})=\mathrm{V} 2 / \mathrm{R}$. As a result, with the increase in the external load resistance, the voltage output (red) increases and the power output (blue) forms an optimum value. It can be noted that the Y-TENG generates a maximum power of $18.22 \mu \mathrm{W}$ at an external load resistance of $10 \mathrm{M} \Omega$.

\section{Conclusions}

We developed a Y-TENG, which is the first report on a TENG based on the Yo-Yo concept. Each generator of the Y-TENG is composed of a case, shaft string, and blade inside the case. A PTFE layer and Al electrode layer are attached on both sides of the blade and the inner surfaces of the case, respectively. When the Y-TENG is released by snap throwing force and gravity, the relative motion between the case and the blade generates electrical output. One generator of Y-TENG produces a maximum $V_{O C}$ of $10 \mathrm{~V}$ and a maximum $I_{C C}$ of $0.7 \mu \mathrm{A}$. The electrical output was measured in terms of the peak and RMS voltage depending on the number of wings and dielectric material. The highest $V$ RMS output was generated when the blade had four wings and when the dielectric layer on the blade 
was PTFE. The proposed Y-TENG continuously lit up three LEDs as the Y-TENG dropped and rose.

Supplementary Materials: The following are available online at https:/ / www.mdpi.com/1996-107 3/14/7/1798/s1, Figure S1: Mechanical analysis title, Figure S2: Mechanical behavior of the blade depending on number of wings, Figure S3: Photographs of the fabricated Y-TENG, Figure S4: Voltage and power output according to external load resistances.

Author Contributions: D.H. and J.C. designed experiments and wrote the manuscript. G.S., M.S. and C.L. performed experiments. D.H. and J.C. analyzed the data. S.L. provided expertise and feedback. All authors have read and agreed to the published version of the manuscript.

Funding: This research was supported by the Chung-Ang University Research Scholarship Grants in 2019 and National Research Foundation of Korea (NRF) grant funded by the Korea government (MSIT) (No.2020R1A2C1010829).

Institutional Review Board Statement: Not applicable.

Informed Consent Statement: Not applicable.

Data Availability Statement: Not applicable.

Conflicts of Interest: The authors declare no conflict of interest.

\section{References}

1. Lee, G.; Kim, M.-C.; Choi, Y.W.; Ahn, N.; Jang, J.; Yoon, J.; Kim, S.M.; Lee, J.-G.; Kang, D.; Jung, H.S.; et al. Ultra-flexible perovskite solar cells with crumpling durability: Toward a wearable power source. Energy Environ. Sci. 2019, 12, 3182-3191. [CrossRef]

2. Park, T.; Na, J.; Kim, B.; Kim, Y.; Shin, H.; Kim, E. Photothermally Activated Pyroelectric Polymer Films for Harvesting of Solar Heat with a Hybrid Energy Cell Structure. ACS Nano 2015, 9, 11830-11839. [CrossRef] [PubMed]

3. Sa, C.; Xu, X.; Wu, X.; Chen, J.; Zuo, C.; Fang, X. A wearable helical organic-inorganic photodetector with thermoelectric generators as the power source. J. Mater. Chem. C 2019, 7, 13097-13103. [CrossRef]

4. Xia, C.; Zhang, D.; Pedrycz, W.; Fan, K.; Guo, Y. Human Body Heat Based Thermoelectric Harvester with Ultra-Low Input Power Management System for Wireless Sensors Powering. Energies 2019, 12, 3942. [CrossRef]

5. Kuhn, V.; Lahuec, C.; Seguin, F.; Person, C. A Multi-Band Stacked RF Energy Harvester With RF-to-DC Efficiency Up to 84\%. IEEE Trans. Microw. Theory Tech. 2015, 63, 1768-1778. [CrossRef]

6. Leon-Gil, J.A.; Cortes-Loredo, A.; Fabian-Mijangos, A.; Martinez-Flores, J.J.; Tovar-Padilla, M.; Cardona-Castro, M.A.; MoralesSánchez, A.; Alvarez-Quintana, J. Medium and Short Wave RF Energy Harvester for Powering Wireless Sensor Networks. Sensors 2018, 18, 768. [CrossRef] [PubMed]

7. $\mathrm{Hu}, \mathrm{Y}$; Wang, Z.L. Recent progress in piezoelectric nanogenerators as a sustainable power source in self-powered systems and active sensors. Nano Energy 2015, 14, 3-14. [CrossRef]

8. Xu, S.; Hansen, B.J.; Wang, Z.L. Piezoelectric-nanowire-enabled power source for driving wireless microelectronics. Nat. Commun. 2010, 1, 93. [CrossRef] [PubMed]

9. Liu, H.; Hou, C.; Lin, J.; Li, Y.; Shi, Q.; Chen, T.; Sun, L.; Lee, C. A non-resonant rotational electromagnetic energy harvester for low-frequency and irregular human motion. Appl. Phys. Lett. 2018, 113, 203901. [CrossRef]

10. Rahman, M.T.; Rana, S.S.; Maharjan, P.; Bhatta, T.; Park, J.Y. Biomechanical Energy-Driven Hybridized Generator as a Universal Portable Power Source for Smart/Wearable Electronics. Adv. Energy Mater. 2020, 10, 1903663. [CrossRef]

11. Choi, J.H.; Cha, K.J.; Ra, Y.; La, M.; Park, S.J.; Choi, D. Development of a triboelectric nanogenerator with enhanced electrical output performance by embedding electrically charged microparticles. Funct. Compos. Struct. 2019, 1, 045005. [CrossRef]

12. Ra, Y.; Choi, J.H.; La, M.; Park, S.J.; Choi, D. Development of a highly transparent and flexible touch sensor based on triboelectric effect. Funct. Compos. Struct. 2019, 1, 045001. [CrossRef]

13. Shi, J.; Chen, X.; Li, G.; Sun, N.; Jiang, H.; Bao, D.; Xie, L.; Peng, M.; Liu, Y.; Wen, Z.; et al. A liquid PEDOT: PSS electrode-based stretchable triboelectric nanogenerator for a portable self-charging power source. Nanoscale 2019, 11, 7513-7519. [CrossRef] [PubMed]

14. Wang, S.; Lin, L.; Wang, Z.L. Nanoscale Triboelectric-Effect-Enabled Energy Conversion for Sustainably Powering Portable Electronics. Nano Lett. 2012, 12, 6339-6346. [CrossRef]

15. Heo, D.; Chung, J.; Kim, B.; Yong, H.; Shin, G.; Cho, J.-W.; Kim, D.; Lee, S. Triboelectric speed bump as a self-powered automobile warning and velocity sensor. Nano Energy 2020, 72, 104719. [CrossRef]

16. Liu, X.; Yu, A.; Qin, A.; Zhai, J. Highly Integrated Triboelectric Nanogenerator for Efficiently Harvesting Raindrop Energy. Adv. Mater. Technol. 2019, 4, 1900608. [CrossRef]

17. Liu, D.; Yin, X.; Guo, H.; Zhou, L.; Li, X.; Zhang, C.; Wang, J.; Wang, Z.L. A constant current triboelectric nanogenerator arising from electrostatic breakdown. Sci. Adv. 2019, 5, eaav6437. [CrossRef] 
18. Zhang, H.; Xia, K.; Fu, J. Pinching a triboelectric nanogenerator using soft pottery for powering electronics. Smart Mater. Struct. 2019, 28, 085036. [CrossRef]

19. Zhang, H.; Yang, Y.; Zhong, X.; Su, Y.; Zhou, Y.; Hu, C.; Wang, Z.L. Single-Electrode-Based Rotating Triboelectric Nanogenerator for Harvesting Energy from Tires. ACS Nano 2014, 8, 680-689. [CrossRef]

20. Zhang, N.; Qin, C.; Feng, T.; Li, J.; Yang, Z.; Sun, X.; Liang, E.; Mao, Y.; Wang, X. Non-contact cylindrical rotating triboelectric nanogenerator for harvesting kinetic energy from hydraulics. Nano Res. 2020, 13, 1903-1907. [CrossRef]

21. Kim, B.; Chung, J.; Moon, H.; Kim, D.; Lee, S. Elastic spiral triboelectric nanogenerator as a self-charging case for portable electronics. Nano Energy 2018, 50, 133-139. [CrossRef]

22. Moon, H.; Chung, J.; Kim, B.; Yong, H.; Kim, T.; Lee, S.; Lee, S. Stack/flutter-driven self-retracting triboelectric nanogenerator for portable electronics. Nano Energy 2017, 31, 525-532. [CrossRef]

23. Chung, J.; Yong, H.; Moon, H.; Van Duong, Q.; Choi, S.T.; Kim, D.; Lee, S. Hand-Driven Gyroscopic Hybrid Nanogenerator for Recharging Portable Devices. Adv. Sci. 2018, 5, 1801054. [CrossRef]

24. Tang, Q.; Yeh, M.-H.; Liu, G.; Li, S.; Chen, J.; Bai, Y.; Feng, L.; Lai, M.; Ho, K.-C.; Guo, H.; et al. Whirligig-inspired triboelectric nanogenerator with ultrahigh specific output as reliable portable instant power supply for personal health monitoring devices. Nano Energy 2018, 47, 74-80. [CrossRef]

25. Wang, S.; Xie, Y.; Niu, S.; Lin, L.; Wang, Z.L. Freestanding Triboelectric-Layer-Based Nanogenerators for Harvesting Energy from a Moving Object or Human Motion in Contact and Non-contact Modes. Adv. Mater. 2014, 26, 2818-2824. [CrossRef]

26. Mao, Y.; Geng, D.; Liang, E.; Wang, X. Single-electrode triboelectric nanogenerator for scavenging friction energy from rolling tires. Nano Energy 2015, 15, 227-234. [CrossRef]

27. Zhang, C.; Zhou, T.; Tang, W.; Han, C.; Zhang, L.; Wang, Z.L. Rotating-Disk-Based Direct-Current Triboelectric Nanogenerator. Adv. Energy Mater. 2014, 4, 1301798. [CrossRef]

28. Zou, H.; Zhang, Y.; Guo, L.; Wang, P.; He, X.; Dai, G.; Zheng, H.; Chen, C.; Wang, A.C.; Xu, C.; et al. Quantifying the triboelectric series. Nat. Commun. 2019, 10, 1-9. [CrossRef]

29. Wang, Z.L. Triboelectric Nanogenerators as New Energy Technology for Self-Powered Systems and as Active Mechanical and Chemical Sensors. ACS Nano 2013, 7, 9533-9557. [CrossRef] 